\title{
Combined Angiotensin Receptor Blocker Losartan and the CXCR4 Inhibitor AMD3100 Increases the Efficacy of Radiotherapy in a Metastatic Osteosarcoma Mouse Model
}

\author{
Sen $\mathrm{Li}^{1,2}$, Wende Li ${ }^{1,3}$, Chi-Ho Leung ${ }^{1,4}$, Shuji Kitahara ${ }^{1,5}$, Yujiao Liü ${ }^{1}$, Sebastian Klein ${ }^{1,6}$, Dai Fukumura ${ }^{1}$, Leo E Gerweck ${ }^{1}$, Jay S Loeffler ${ }^{1}$ \\ Rakesh K Jain ${ }^{1}$, Dan G Duda ${ }^{1 * \#}$ and Peigen Huang ${ }^{1 * \#}$ \\ ${ }^{1}$ Department of Radiation Oncology, Massachusetts General Hospital, Harvard Medical School, Boston, MA USA \\ ${ }^{2}$ Department of Spinal Surgery, Affiliated Traditional Chinese Medicine Hospital, Southwest Medical University. Luzhou, P.R. China \\ ${ }^{3}$ Guangdong Laboratory Animal Monitoring Institute, Guangzhou, P.R. China \\ ${ }^{4}$ Division of Urology, Department of Surgery, Prince of Wales Hospital, The Chinese University of Hong Kong, Shatin, Hong Kong \\ ${ }^{5}$ Department of Anatomy and Developmental Biology, School of Medicine, Tokyo Women's Medical University, Tokyo, Japan \\ ${ }^{6}$ Institute for Pathology, University Hospital of Cologne, Cologne, Germany \\ \#Contributed equally to the paper
}

\begin{abstract}
Objective: Osteosarcoma (OS) is highly metastatic and the most common primary malignant bone tumor. Hypoxia and CXCR4-overexpression in OS may play a role in resistance to radiotherapy. Using a metastatic OS mouse model, we investigated whether combining radiotherapy with a stroma-modifying drug (the angiotensin receptor 1 blocker losartan) and an anti-metastatic agent (the CXCR4 inhibitor AMD3100) is an effective OS treatment strategy.
\end{abstract}

Material and Methods: A highly metastatic, CXCR4-overexpressing Os-P0107 cell line was used to generate subcutaneous isografts in syngeneic $\mathrm{C} 3 \mathrm{Hf} / \mathrm{Sed}$ mice. When the tumors reached $6 \mathrm{~mm}$ in diameter, we treated the mice with either losartan (40 mg/kg body weight, gavage), AMD3100 (AMD, $5 \mathrm{mg} / \mathrm{kg}$ body weight, i.p.), or a combination of both drugs daily for 14 days, with $20 \mathrm{~Gy}$ local irradiation (IR) on day 7 . We evaluated the tumorgrowth delay (TGD), distant metastases and host survival, as well as tumor vascular perfusion and tumor hypoxia.

Results: Treatment with IR, Losartan+IR, or AMD+IR resulted in a significant and comparable TGD (12 to 20 days) in Os-P0107 tumors versus the controls (all $p<0.01$ ). However, only the combination of Losartan+AMD+IR significantly enhanced tumor response to radiation by increasing TGD (additional 12 days, Losartan+AMD+IR vs. IR $p=0.0215)$, decreasing distant metastasis $(p=0.008)$, and increasing survival $(p=0.025)$. Losartan treatment significantly increased CD31 positive tumor vascular density and decreased pimonidazole positive (hypoxic) areas (Percentage of CD31 and Pimo positive area; Losartan vs. Control, both $\mathrm{p}<0.01$ ).

Conclusion: The combination of Losartan+AMD+IR significantly increases the efficacy of radiotherapy in a highly metastatic OS mouse model. The therapeutic effects are most likely due to the targeting both of tumor hypoxia and CXCR4 by losartan and AMD3100 with local irradiation. Our finding strongly suggests that losartan and AMD3100 with radiotherapy could be a potential new strategy for clinical metastatic OS treatment.

Keywords: Radiotherapy; Angiotensin 1 receptor; CXCR4; Metastatic osteosarcoma; Mouse model

Abbreviations: OS: Osteosarcoma; BW: Body Weight; IR: Irradiation; TGD: Tumor-Growth Delay; CAFs: Cancer Associated Fibroblasts; DMEM: Dulbecco's Modified Eagle's Medium; FBS: Fetal Bovine Serum; PE: Plating Efficiency; SF: Surviving Fraction; qRT-PCR: Quantitative Reverse Transcription Polymerase Chain Reaction; DAB: Diaminobenzidine; MFP: Mammary Fat Pad

\section{Introduction}

Osteosarcoma (OS) is the most common primary malignant bone tumor that often occurs in children and young adults [1-4]. Most OS are high grade, very aggressive, and tend to rapidly develop distant metastases [3-6]. OS is commonly considered a radioresistant tumor, thus experience with radiotherapy in the treatment of OS is limited. However, recent data suggests that the administration of radiotherapy may be useful in patients treated with multi-agent chemotherapy who are unable to undergo complete resection or who have microscopic residual tumor foci following attempted resection [7]. Increased tumor hypoxia and the expression of the pro-metastatic receptor CXCR4 are both significantly elevated in human metastatic OS and have been shown to be associated with resistance to radiation therapy and poor clinical outcome [5,8-14]. Thus, developing approaches to reduce hypoxia and to target CXCR4 and combining them with radiation therapy are attractive strategies for improving treatment response in metastatic OS [2,6,7,14-16]. AMD3100, a small molecular weight drug that inhibits CXCR4 has been shown to decrease the in vitro and in vivo migration and invasion of OS and other cancer cells [17-21]. AMD3100 can also enhance tumor radio-sensitivity as reported in multiple cancer mouse models $[22,25]$. Unfortunately, highly spontaneous metastatic OS animal

*Corresponding author: Peigen Huang, Department of Radiation Oncology, Massachusetts General Hospital, 100 Blossom Street, Cox-7, Boston, MA 02114, USA Tel: (617) 726-8143; Fax: (617) 724-5841; E-mail: peigen@steele.mgh.harvard.edu

Dr. Dan G. Duda, Department of Radiation Oncology, Massachusetts General Hospital, 100 Blossom Street, Cox-7, Boston, MA 02114, USA, Tel: (617) 726-8143 Fax: (617) 724-5841; E-mail: Mduda@steele.mgh.harvard.edu

Received October 31, 2018; Accepted December 07, 2018; Published December 10, 2018

Citation: Li S, Li W, Leung C, Kitahara S, Liu Y, et al. (2018) Combined Angiotensin Receptor Blocker Losartan and the CXCR4 Inhibitor AMD3100 Increases the Efficacy of Radiotherapy in a Metastatic Osteosarcoma Mouse Model. J Cancer Sci Ther 10: 371-378. doi: 10.4172/1948-5956.1000570

Copyright: () $2018 \mathrm{Li} \mathrm{S}$, et al. This is an open-access article distributed unde the terms of the Creative Commons Attribution License, which permits unrestricted use, distribution, and reproduction in any medium, provided the original author and source are credited. 
Citation: Li S, Li W, Leung C, Kitahara S, Liu Y, et al. (2018) Combined Angiotensin Receptor Blocker Losartan and the CXCR4 Inhibitor AMD3100 Increases the Efficacy of Radiotherapy in a Metastatic Osteosarcoma Mouse Model. J Cancer Sci Ther 10: 371-378. doi: 10.4172/19485956.1000570

models with CXCR4 expression are lacking [26,27], making it difficult to study the efficacy of targeting CXCR4 with radiotherapy in OS.

We have established a novel OS tumor line (Os-P0107) exhibiting high in vivo tumorigenicity, transplant ability, and potential for spontaneous metastasis to multiple host organs after primary tumor resection [28]. Our recent studies showed that Os-P0107 cells are highly resistant to radiation in vitro, resembling the clinical situation. We detected high CXCR4 expression in primary, locally recurrent, and metastatic Os-P0107 tumor tissues. Moreover, these tumor tissues exhibited a high collagen density stroma. Thus, this highly metastatic Os-P0107 tumor line may serve as a useful in vivo model to study new therapeutic strategies targeting tumor hypoxia and CXCR4 in metastatic OS.

We previously found that cancer cells and cancer-associated fibroblasts (CAFs) actively exert physical forces (solid tress) to compress tumor blood vessels, thus reducing vascular perfusion [29]. These cellular components store and transmit the solid stress through the interstitial matrix molecules collagen and hyaluronan, making both CAFs and matrix critical targets for decompressing tumor vessels [30-32]. We have reported that losartan - a drug commonly used in the clinic to treat hypertension - improves the outcome of cancer treatment by decompressing collapsed tumor blood vessels to increase tumor perfusion in both breast and pancreatic cancer models [30-32]. Losartan, through its activity as an angiotensin II receptor 1 antagonist, deactivates CAFs and thus reduces the expression of the profibrotic signals TGF- $\beta$, CCN2, and ET-1, leading to a reduction in stromal collagen and hyaluronan production $[33,34]$. As a result, losartan can reduce solid stress and increase vascular perfusion in tumors. Through this physical mechanism, losartan can improve drug and oxygen delivery to tumors [30-32]. Here, we evaluated the effect of combining losartan and AMD3100 with ionizing radiation in a collagen rich and high CXCR4-expressing Os-P0107 metastatic tumor model by measuring local tumor response, distant metastases and host survival, as well as tumor vascular perfusion and tumor hypoxia. Our results show that combining losartan and AMD3100 with radiotherapy not only significantly enhances Os-P0107 isograft tumor-gowth delay, but also decreases metastatic burden and prolongs the survival of host mice.

\section{Materials and Methods}

\section{Cell culture and proliferation assay}

The Os-P0107 tumor line used in the present study was established in our laboratory as previously reported [28]. The cell line was routinely cultured and maintained in $25 \mathrm{~cm}^{2}$ tissue culture flasks containing $5 \mathrm{ml}$ of Dulbecco's modified Eagle's medium (DMEM) supplemented with $10 \%$ heat-inactivated fetal bovine serum (FBS; Gibco, Carlsbad, CA, USA), incubated in $5 \% \mathrm{CO}_{2}$ at $37^{\circ} \mathrm{C}$. For colony forming efficiency assay, Os-P0107 cells were plated into 5 T-25 flasks with 100, 200, 400, 600 , and 800 cells respectively, and cultured in DMEM supplemented with 13\% FBS in incubator for 12 days. Then colonies (formed with 50 or greater cells) were fixed, stained and counted. The plating efficiency (PE) was calculated by the formula: $\mathrm{PE}=$ colony number/cell number $\mathrm{x}$ $100 \%$. All in vitro experiments were repeated at least three times.

\section{In vitro colony formation assay}

The colony formation assay was used to examine the therapeutic effects of radiation, AMD3100, or losartan on Os-P0107 cells in vitro. For radiation effects, a single cell suspension of the cells was prepared, counted, plated into culture flasks $\left(25 \mathrm{~cm}^{2}\right)$, and irradiated $20-24$ hours later with $320-\mathrm{kV}, 12.5 \mathrm{~mA}, \mathrm{X}$-ray at a nominal dose rate of $1.67 \mathrm{~Gy}$ per minute ( 6 doses: 0 Gy to $10 \mathrm{~Gy}$ ). The surviving fraction (SF) data were corrected for initial and final multiplicity determined 4 to 6 hours after plating and at the time of irradiation [35]. At each dose point, 5 flasks containing a 2-4-fold range of cell numbers were plated. Following irradiation, the cells were routinely cultured for 12 (for control cells) to 14 days (for cells exposed to high doses of radiation), i.e., when the number of colonies (formed with 50 or greater cells) per flask did not change. The colonies were then fixed, stained and counted. The cell SFs were calculated by the formula: $\mathrm{SF}=$ (number of colonies/number of cells plated)/PE.

For losartan or AMD3100 therapeutic effects, a single cell suspension with different number of cells was cultured in DMEM supplemented with 15\% FBS and different concentrations of losartan or AMD3100 for 10 to 14 days; then the colony formation assay was performed. A single dose of AMD3100 combined with radiation treatment was also tested by in vitro colony formation assay using the above methods.

\section{Animal and in vivo tumor growth}

Six to eight weeks old male $\mathrm{C} 3 \mathrm{Hf} / \mathrm{Sed}(\mathrm{C} 3 \mathrm{H})$ mice were used in our study. All mice were bred and maintained in our Cox-7 defined flora animal facility [36], and experimental mice were housed in microisolator cages, fed with sterile laboratory pellets, and given acidified sterile water ad libitum. All animal care and studying procedures were carried out following the Public Health Service Policy on Humane Care of Laboratory Animals and approved by the Institution Animal Care and Use Committee at the Massachusetts General Hospital.

To generate the source tumors of the Os-P0107 tumor line in vivo (which were initiated from in vitro cell cultures), approximately $10^{5}$ tumor cells (in $0.5 \mathrm{ml}$ PBS) were injected into the subcutaneous (s.c.) tissue of $\mathrm{C} 3 \mathrm{H}$ mice in their right hind limbs. Mice then were observed weekly for tumor development and growth. The tumor "take" rate of Os- $\mathrm{P} 0107$ in $\mathrm{C} 3 \mathrm{H}$ mice was 80 to $100 \%$ as before [28].

\section{Tumor responses to combining radiotherapy}

Small (1-2 mm diameter) fragments from Os-P0107 source tumors (in vivo $\mathrm{F} 2$ to $\mathrm{F} 4$ passages of isografts) were implanted s.c. into the right hind limb of $\mathrm{C} 3 \mathrm{H}$ mice (10-20 mice/each group). A single source tumor from each passage was used to initiate all tumors for each experimental cohort. After implantation, two perpendicular diameters of the tumors were measured once to twice per week with calipers. Tumor volume $(V)$ was calculated as $V=a \times b^{2} / 2$, where $a$ and $b$ are the long and short axes, respectively [28]. When the isografted tumors reached a size of 5 $\times 6 \mathrm{~mm}$ in diameter (or approximately $75 \mathrm{~mm}^{3}$ ), they were randomly assigned to control or treatment groups. Experimental mice were treated for 14 days with either Losartan, $40 \mathrm{mg} / \mathrm{kg}$ body weight (BW) or PBS $0.2 \mathrm{ml}$ as a control by oral gavage daily with and without a 20 Gy single dose local irradiation (IR) on day7; Alternatively, mice were treated for 14 days with AMD3100, $5 \mathrm{mg} / \mathrm{kg}$ BW (AMD) or PBS $0.2 \mathrm{ml}$ as a control by i.p. injection once a day with and without a 20 Gy IR on day 7. In addition, one arm of the animals received triple combination treatment of Losartan+AMD+IR. Single dose (20 Gy) irradiation (320$\mathrm{kV}, 12.5 \mathrm{~mA}, 3.76 \mathrm{~Gy}$ per minute) was given under normal blood flow conditions utilizing a $1 \mathrm{~cm}$ field centered over the tumor; then the tumor-growth delay (TGD) was determined.

\section{Metastasis and survival assay}

To determine their metastatic frequency, the isografted primary tumors in the right hind-limbs of mice were removed. When the primary tumors reached a size of $13 \mathrm{~mm} \times 13 \mathrm{~mm}$ (or approximately $\left.1000 \mathrm{~mm}^{3}\right)$, the mice were anesthetized $(90 \mathrm{mg} / \mathrm{kg}$ Ketamine and $9 \mathrm{mg}$ / 
Citation: Li S, Li W, Leung C, Kitahara S, Liu Y, et al. (2018) Combined Angiotensin Receptor Blocker Losartan and the CXCR4 Inhibitor AMD3100 Increases the Efficacy of Radiotherapy in a Metastatic Osteosarcoma Mouse Model. J Cancer Sci Ther 10: 371-378. doi: 10.4172/19485956.1000570

$\mathrm{kg}$ Xylazine, i.p.), and the tumor-bearing legs were sterilized using $70 \%$ alcohol. The blood flow was stopped by means of a clamp attached at mid-thigh for 5 minutes, and the tumor bearing legs then were amputated. Any bleeding was controlled, and the resulting wound was closed with a 9-mm wound autoclip (Clay Adams, Division of Becton Dickinson and Co., Parsippany, NJ), the wound clips were removed 2 weeks later. The surgical procedure was described previously [37]. Post-operative analgesia was supplied by administering buprenorphine $(0.1 \mathrm{mg} / \mathrm{kg}$ s.c. $\mathrm{q} 12$ hours $)$ for 3 days. The first dose of buprenorphine was administered about 30 minutes pre-procedurally as a pre-emptive analgesia for post-operative pain control. All the mice with limb removal were weighted at one day post amputation for future comparison of body weight changes. In our experience, mice undergoing such a procedure could fully ambulate within two days of the procedure and could reach a normal active condition compared to the un-amputated mice at one-week post-operation. The mice were euthanized 3 months after primary tumor implantation or when they became moribund, whichever came first. Gross autopsy and histopathological analyses were performed then. Survival experiments were terminated when mice became moribund, or lost more than $15 \%$ of body weight, or reached the end-point.

\section{Quantitative reverse transcription polymerase chain reaction (qRT-PCR)}

qRT-PCR was performed to test CXCR4 expression in Os-P0107 cells, and in primary and metastatic tumors. In brief, total RNA was extracted from Os-P0107 cells and fresh tumor tissues using an RNeasy Mini Kit (QIAGEN). cDNAs were synthesized using the TaqMan RT Kit (Applied Biosystems, Branchburg, NJ). Primers specific for $\beta$-actin and CXCR4 were used, and relative gene expression was determined using Real-Time SYBR Green PCR Master Mix (Applied Biosystems) on a Stratagene Mx3000P qPCR System, as described [38].

\section{Immunohistochemistry and Immunofluorescence}

To evaluate CXCR4 expression in Os-P0107 tumors, the polyclonal rabbit anti-CXCR4 antibody was used (dilution 1:100). Five-micrometer sections of formalin-fixed paraffin-embedded tumor tissues were mounted on Poly-L-lysinecoated slides. The slides were air dried and the tissue was deparaffinized. Endogenous peroxidase activity was blocked with $3 \%$ hydrogen peroxide in $50 \%$ methanol for 10 minutes at room temperature. The sections were rehydrated and washed with PBS and then pretreated with citrate buffer $(0.01$ $\mathrm{M}$ citric acid, $\mathrm{pH}$ 6.0) for 15 minutes at $97^{\circ} \mathrm{C}$ in a microwave oven. After nonspecific binding sites were blocked by incubating slides in $2 \%$ normal goat serum in PBS for 15 minutes at room temperature, the sections were incubated overnight at $4^{\circ} \mathrm{C}$ with the primary rabbit anti-CXCR4 antibody. The sections were then washed with PBS and incubated with the secondary HRP-conjugated antibody for 30 minutes at room temoerature. Staining was performed by incubating slides with fresh 3,3'diaminobenzidine (DAB) buffer. The sections were washed in running water and counterstained with hematoxylin, followed by dehydration and mounting. PBS was utilized in place of the primary antibody for the negative control.

For evaluation of tumor vascular density, frozen tumor sections (7-8 micrometer thick) were immuno-stained with primary antiCD31 (for endothelial cells) antibody and counter stained with DAPI. Samples were imaged by using an Olympus confocal microscope. Pimonidazole (Hypoxyprobe; Hypoxyprobe, Inc.) was used as a marker of tumor hypoxia. Hypoxyprobe $(60 \mathrm{mg} / \mathrm{kg} \mathrm{BW})$ was injected i.p. 30 minutes before the mice were euthanized. Tumors were harvested and processed immediately. Hypoxia was assessed in frozen tissue sections by immunofluorescence staining of pimonidazole by antiHypoxyprobe-fluorescein isothiocyanate (FITC)-labeled antibody, as described [20,38].

\section{Statistical analysis}

Unless otherwise noted, data are presented as mean \pm SEM. The one-way ANOVA method followed by the Dunnett's post hoc test, and the Unpaired t test (two-tailed) was used to compare the tumor volumes and the days of tumor-growth delay of the treatment groups against the control. Difference in tumor metastatic rates was analyzed by Kendall's tau with the Holm-Bonferroni adjustment. Host survival times (curves) were compared with Gehan-Breslow-Wilcoxon test. All statistical analyses were performed using GraphPad Prism 7 and SPSS version 24 . A difference was considered statistically significant when the $P$ value was less than 0.05 .

\section{Results}

\section{Os-P0107 cells are resistant to radiation in vitro and express higher levels of CXCR4 both in vitro and in vivo}

The cultured Os-P0107 cell line maintained its original characters as previously reported [28] (Supplemental Figure 1). The data are well fit by the linear-quadratic cell survival model, i.e., alpha $=0.160$ $\mathrm{Gy}^{-1}$, beta $=0.023 \mathrm{~Gy}^{-2}, \mathrm{R}^{2}=0.998$. Similar to previous reports, the surviving fractions of Os-P0107 cells at 2 and 10 Gy are 0.662 and 0.198 respectively are substantially more resistant to radiation than most other cultured cell types [39] (Figure 1A). Neither the CXCR4 inhibitor AMD3100 nor the Angiotensin 1 receptor blocker losartan alone showed any significant effect on the colony forming efficiency of this cell line (Figures 1B and 1C). In addition, AMD3100 also did not alter the in vitro radiosensitivity of Os-P0107 cells (Figure 1D). Figure 1D shows that both Os-P0107 cells and tumors (primary and metastatic) expressed high levels of CXCR4 mRNA as evaluated by qRT-PCR. This finding has been confirmed at protein level by Immunohistochemistry staining in the tumor tissues confirmed abundant presence of CXCR4 protein (Figure 1E).

\section{Combination treatment with losartan and AMD3100 significantly increases Os-P0107 tumor-growth delay by radiotherapy}

As shown in Figure 2, a single dose of 20 Gy irradiation (IR) exhibited a significant TGD in Os-P0107 tumors ( 13 days more required to reach the tumor volume of $500 \mathrm{~mm}^{3}$ ) as compared to the control and the losartan or AMD3100 monotherapy groups (IR vs. Control, and IR vs. Losartan, or IR vs. AMD, all $p<0.01$; Losartan $v s$. Control, or AMD vs. Control, both $p>0.05)$. Combined Losartan+IR or AMD+IR treatment did show an improvement in TGD ( $\sim 18$ to 20 days more required to reach the tumor volume of $500 \mathrm{~mm}^{3}$ ), although their comparable TGDs to IR alone did not increased significantly (Losartan+IR, or AMD+IR vs. IR group, both $p>0.05$ ). These results show that losartan or AMD3100 combined with IR did not significantly increase tumor response to IR alone in the Os-P0107 isografts in vivo. However, triple combination treatment with Losartan+AMD+IR significantly increased Os-P0107 TGD by 12 days compared to IR alone (Figure $2 \mathrm{C}, p<0.01$ ).

Combining losartan and AMD3100 with radiotherapy significantly improves the treatment of metastatic Os-P0107 isograft

When evaluating treatment efficacy in survival experiments, 
Citation: Li S, Li W, Leung C, Kitahara S, Liu Y, et al. (2018) Combined Angiotensin Receptor Blocker Losartan and the CXCR4 Inhibitor AMD3100 Increases the Efficacy of Radiotherapy in a Metastatic Osteosarcoma Mouse Model. J Cancer Sci Ther 10: 371-378. doi: 10.4172/19485956.1000570

$\mathbf{A}$
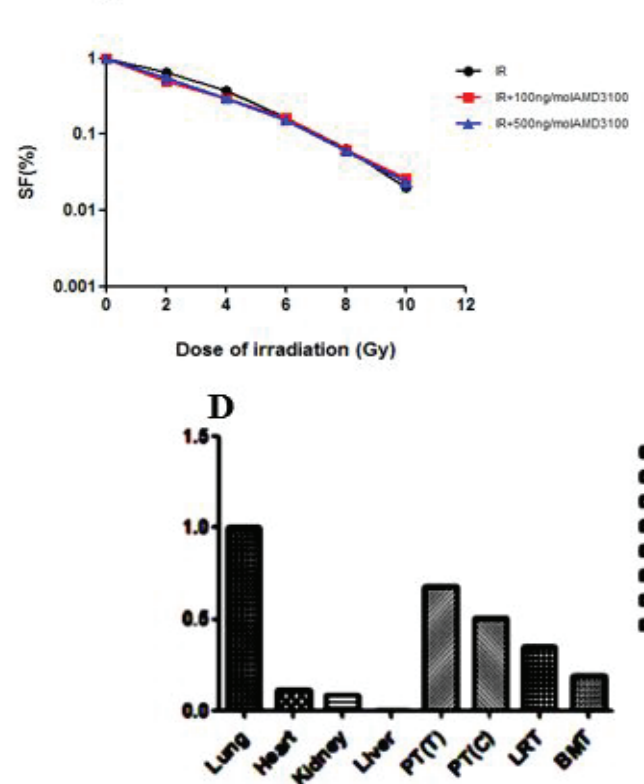

B
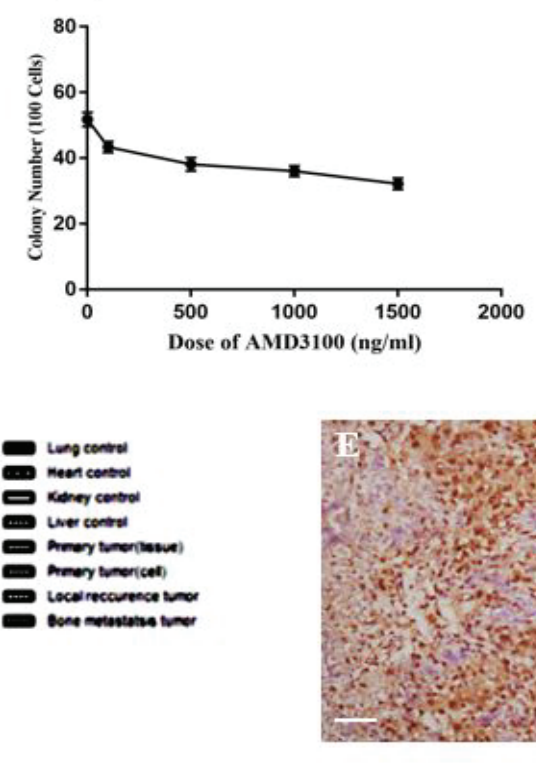

C

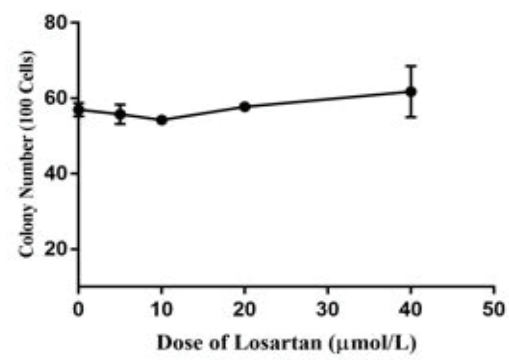

Figure 1: Os-P0107 cell response to treatment in vitro and their CXCR4 expression in vivo. (A-C) Cell survival curves from the colony formation assay show OsP0107 cell response to irradiation and irradiation combined with AMD3100, AMD3100 or losartan alone treatments in vitro. Panel C shows the absence of losartan cytotoxicity at doses up to $40 \mu \mathrm{mol} / \mathrm{L}$; thus, losartan in vitro alone did not play an anti-Os-P0107 cell effect. (D) Higher CXCR4 expression in Os-P0107 tumor tissues (primary, local recurrent, and metastatic tumors) when compared with the host $\mathrm{C} 3 \mathrm{H}$ mice normal tissues (heart, kidney, and liver, except the lung tissues) by qPCR; (E) CXCR4 expression was confirmed by immunohistochemistry staining in primary isografted Os-P0107 tumor. Bar, $50 \mu \mathrm{m}$.

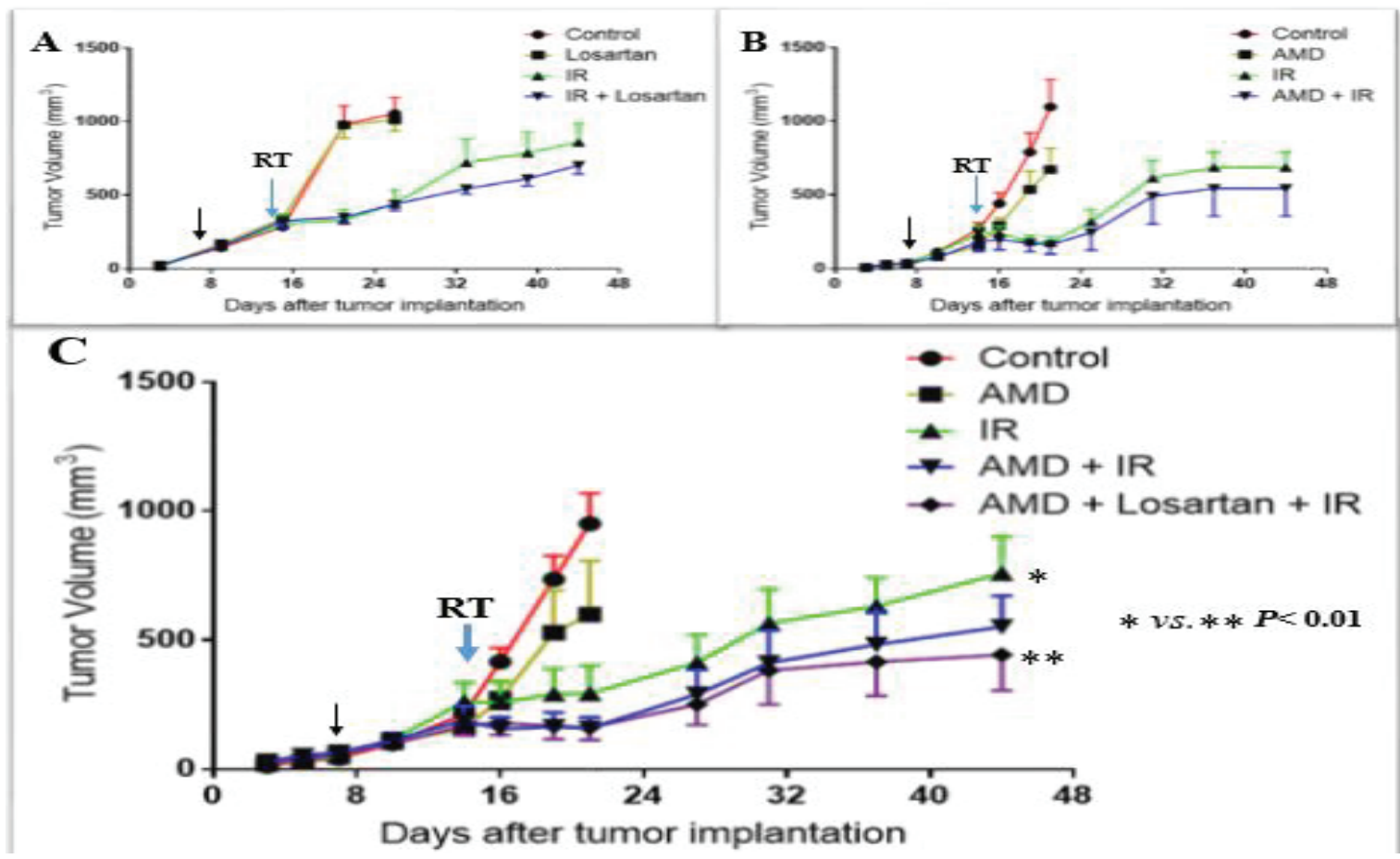

Figure 2: Os-P0107 tumor response to combined losartan, AMD3100, and radiation treatment. (A) Os-P0107 tumors growth and response curves of control, or losartan with/without 20 Gy local irradiation (IR); (B) Tumor growth curves of control, or AMD3100 (AMD) with/without 20 Gy local irradiation; and (C) Tumor growth curves of control, IR, AMD, AMD+IR, and AMD+losartan+IR groups. Results show that IR, Losartan+IR, AMD+IR, or AMD+Losartan+IR treatment could significantly delay tumors growth when compared to control, losartan, or AMD3100 alone groups; but neither losartan nor AMD3100 could significantly improve the Os-P0107 tumors response to radiotherapy (Losartan+IR vs. IR, and AMD+IR vs. IR, both $P>0.05$ ). Only triple combination treatment of $A M D+L o s a r t a n+I R$ can significantly enhance Os-P0107 tumors response to radiation by an additional TGD for 12 days (AMD+Losartan+IR vs. IR, $p=0.0215)$. Data are presented as Mean \pm SEM; $P<0.05$ is considered statistically significant ( $N=10-20 /$ group). 
Citation: Li S, Li W, Leung C, Kitahara S, Liu Y, et al. (2018) Combined Angiotensin Receptor Blocker Losartan and the CXCR4 Inhibitor AMD3100 Increases the Efficacy of Radiotherapy in a Metastatic Osteosarcoma Mouse Model. J Cancer Sci Ther 10: 371-378. doi: 10.4172/19485956.1000570

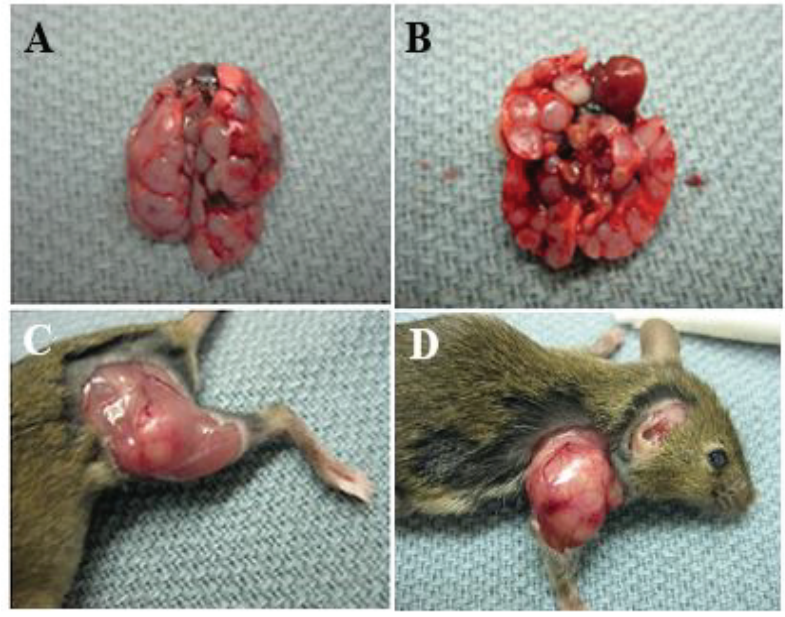

E

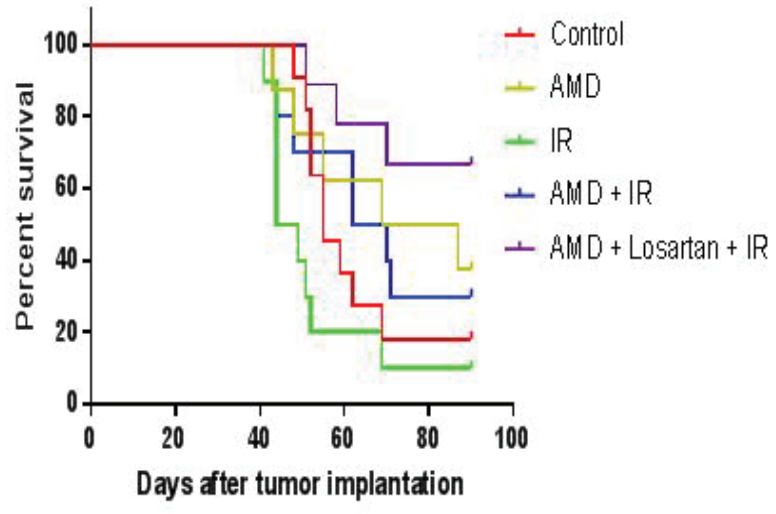

Figure 3: Combining losartan and AMD3100 with radiotherapy decreased Os-P0107 tumor metastasis and increased host survival. (A-D) Photographs of OsP0107 isografts metastases to lungs (A \& B), left leg (C), and humeri (D). (E) Kaplan-Meier survival distribution show that combining losartan and MAD3100 with 20 Gy IR significantly increased host survival (Losartan+AMD+IR vs. Control $p=0.025$; Losartan+AMD+IR vs. IR $p=0.002$ ).
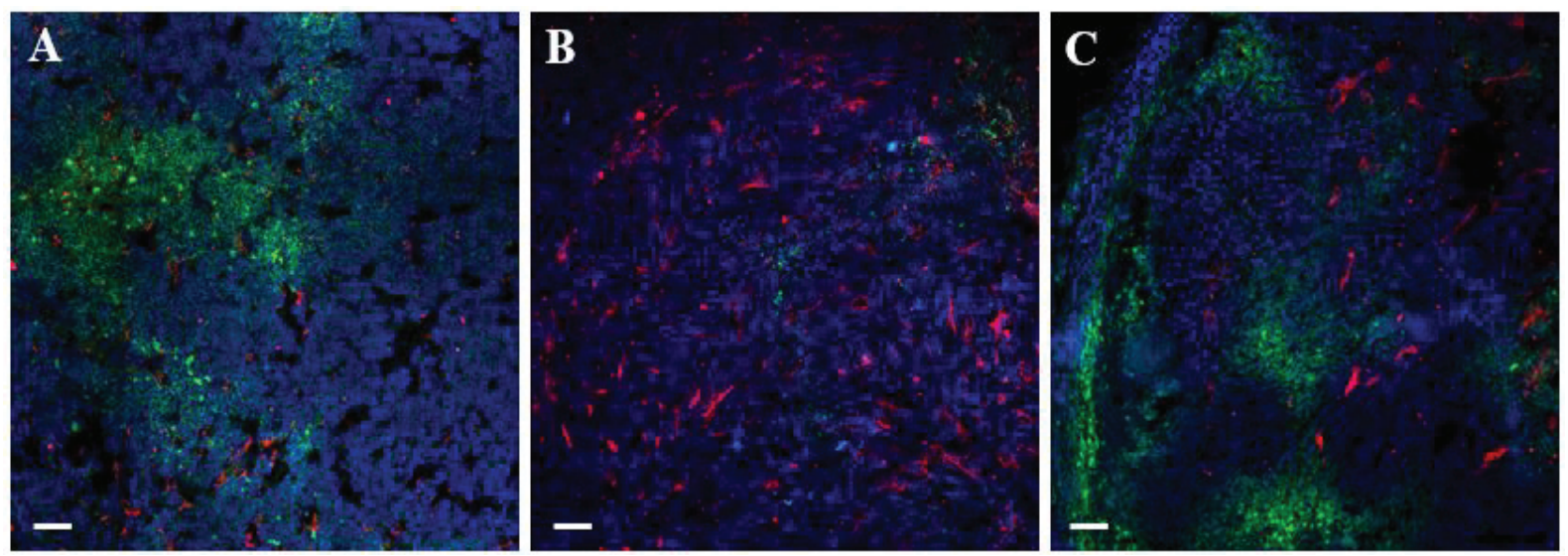

Figure 4: Representative immunohistochemistry of CD31 staining and pimonidazole immunofluorescence in treated and control Os-P0107 tumor tissues. (AC) CD31 positive vascular is shown in red and pimonidazole positive hypoxic area in green. Sections of untreated normal control (A), losartan treated (B), and AMD3100 treated group tumor tissue (C). Bar=50 $\mu \mathrm{m}$.
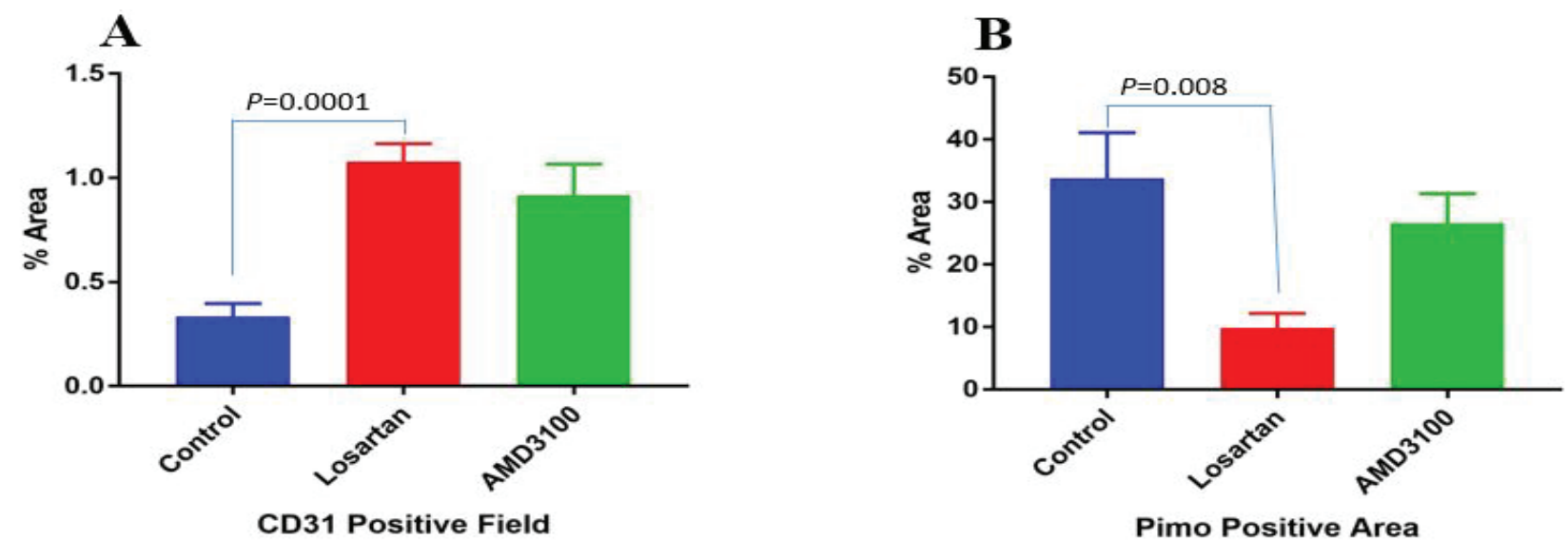

Figure 5: Losartan treatment significantly increased tumor vascular density and decreased tumor hypoxic in Os-P0107 tumor tissues. (A) Losartan treatment significantly increases CD31 positive vascular area (Losartan vs. Control, $p=0.0001$ ). (B) Losartan treatment significantly deceases hypoxia measured as pimonidazole positive area (Losartan vs. Control, $p=0.0003$ ) in Os-P0107 tumor tissues. ( $\mathrm{N}=5-10$ areas/group). 
Citation: Li S, Li W, Leung C, Kitahara S, Liu Y, et al. (2018) Combined Angiotensin Receptor Blocker Losartan and the CXCR4 Inhibitor AMD3100 Increases the Efficacy of Radiotherapy in a Metastatic Osteosarcoma Mouse Model. J Cancer Sci Ther 10: 371-378. doi: 10.4172/19485956.1000570

\begin{tabular}{|c|c|c|c|c|}
\hline \multirow{2}{*}{ Group (N=Mice) } & \multicolumn{4}{|c|}{ Macro Metastases (Lung/Liver/Bone/Kidney) } \\
\hline & Non-Metastasis & One Organ Metastasis & Two/More Organ Metastases & Chi-Square $\left(\mathbf{X}^{2}\right)$ Test \\
\hline "Control $(\mathrm{N}=11)$ & 2 & 1 & 8 & -- \\
\hline${ }^{*} \mathrm{AMD}(\mathrm{N}=8)$ & 3 & 3 & 2 & ${ }^{*} v s^{* *} \quad \mathrm{p}=0.096$ \\
\hline${ }^{* *} I R 20 G y(N=10)$ & 1 & 4 & 5 & ${ }^{*} v s^{*+*} p=0.457$ \\
\hline${ }^{\# A M D+I R ~(N=10)}$ & 3 & 5 & 2 & ${ }^{*} v s^{\#} \quad p=0.093$ \\
\hline${ }^{\# A M D+L o s+I R ~(N=9)}$ & 5 & 3 & 1 & "vs"\# $p=0.008$ \\
\hline
\end{tabular}

Table 1: Combining Losartan and AMD3100 with radiotherapy in metastatic Os-P0107 tumors.

our results showed that triple combination treatment with Losartan+AMD+IR significantly decreased metastatic burden in the OsP0107 tumor model (Losartan+AMD+IR vs. Control group, $p=0.008$; Table 1 and Figures 3A-3D). Furthermore, only Losartan+AMD+IR triple combination treatment significantly increased overall survival (Losartan+AMD+IR vs. Control group, $p=0.025$; Losartan+AMD+IR vs. IR group, $p=0.002$; Figure $3 \mathrm{E}$ ). Thus, combining losartan and AMD3100 with irradiation could significantly delay Os-P0107 tumor growth, and decrease metastases to distant organs, thereby increasing host survival.

\section{Losartan may increase tumor blood perfusion and AMD3100 delivery to Os-P0107 isografts}

Next, we have examined tumor vascular density and tumor hypoxia by CD31 and pimonidazole immunofluorescence staining in control and treated Os-P0107 tumors. We found that losartan treatment significantly increased CD31 positive tumor vascular density and decreased pimonidazole positive (hypoxic) areas (Percentage of CD31 and Pimo positive area: Losartan $v s$. Control, both $p<0.01$; Figures 4 and 5), and AMD3100 treatment alone did not significantly decrease the pimonidazole positive areas (Percentage of Pimo positive area: AMD vs. Control, $p>0.01$; Figure $5 \mathrm{~B}$ ). This result indicates that losartan plus AMD3100 enhanced the Os-P0107 tumors response to radiation treatment possibly by losartan reducing solid stress in these stromal collagen-rich OS isografts, then increasing tumor blood perfusion and improving AMD3100 delivery to tumors.

\section{Discussion}

OS is one of the most common primary high-grade malignant bone tumors with a poor prognosis [1-6]. In the clinic, localized OS is typically treated with surgical resection and chemotherapy; radiotherapy is reserved for rare unresectable cases [40-42]. Distant metastasis is present at diagnosis in approximately $10-20 \%$ of OS patients and develops in $30-50 \%$ of patients who initially present with localized OS. The ten-year survival of OS patients with metastatic disease is approximately $24 \%$ [40]. Multiple organ metastases are the main factors for the clinical failure. Despite numerous attempts in large clinical trials to augment therapy via dose intensification and the addition of chemotherapeutic agents, OS survival rates have stagnated over the past three decades [41,42]. The debilitating side effects of the surgical treatment suggest the need for alternative local control approaches and adding radiotherapy could potentially improve patient outcomes [41-44]. However, OS has been commonly considered to be a low radiosensitive tumor, and few reports have described treatment outcome of OS using radiotherapy, thus the experience with radiotherapy in treatment of OS is limited $[7,43,44]$.

In this study, we first report that combining an angiotensin receptor blocker (losartan) and a CXCR4 inhibitor (AMD3100) with radiotherapy can significantly enhance tumor-growth delay, decrease metastasis, and increase host survival time in a metastatic Os-P0107 mouse model. Os-P0107 is an established and well maintained in vitro and in vivo spontaneous OS mouse model in our laboratory and is highly metastatic in $\mathrm{C} 3 \mathrm{H}$ background transgenic and wild-type mice as previously reported [28]. High levels of CXCR4 expressions have been detected in cultured Os-P0107 cells and in in vivo primary and metastatic tumors. The Os-P0107 tumor is also a stromal collagenrich tumor (Supplemental Figure 2; Dr. Yves Boucher, unpublished data). Tumor and tumor stromal cells actively exert physical forces (solid stress) to compress tumor blood vessels, thus reducing vascular perfusion [29-32]. Our previous studies have demonstrated that losartan could reduce stromal collagen and hyaluronan production, then reduce solid stress in tumors resulting in increased vascular perfusion [31,32]. Thus, losartan could improve drug and oxygen delivery to tumors, thus potentiating chemotherapy and reducing hypoxia in breast and pancreatic cancer models [31].

Here, we report that combining losartan and AMD3100 can significantly enhance radiation response in local Os-P0107 tumors, resulting in a substantial TGD compared to that by irradiation alone (12 days). Recently, there are studies showing losartan is a potential therapeutic agent in cancer treatment [45]. However, our studies show that either losartan nor AMD3100 alone could radiosensitize Os-P0107 cells in vitro or isografted tumors in vivo. Losartan treatment could significantly increase tumor vascular density (CD31 positive vessels) and decrease tumor tissue hypoxia (the pimonidazole positive areas) in Os-P0107 isografts. All these results together may indicate that losartan can increase tumor blood perfusion and improve tumor tissue oxygenation and drug delivery to Os-P0107 tumors by reducing their tumor solid stress, thus enhancing tumor response to radiotherapy and AMD3100.

We have performed studies in another tumor line, MCa-M3C, to test the combination of losartan plus radiation on the tumor response to radiotherapy recently. MCa-M3C is a novel HER2/neu-positive metastatic breast tumor model established and characterized in vitro and in vivo in our laboratory (Unpublished data). Our studies showed that combining losartan with radiotherapy (20 Gy single dose or $5 \mathrm{~Gy}$ $\times 4$ fractions) increased the responses of the MCa-M3C orthotopic mammary fat pad (MFP) tumors. The combination treatment significantly enhanced TGD, decreased MCa-M3C MFP-to-lung metastases, and prolonged host survival. Our studies also showed that losartan significantly increased MCa-M3C MFP tumor vascular volume and reduced tumor hypoxia (Unpublished data). These combination treatment results from the $\mathrm{MCa}-\mathrm{M} 3 \mathrm{C}$ tumor line are consistent with our Os-P0107 studies.

Recent research by others has revealed that tumor hypoxia and high-level expression of the pro-metastatic receptor CXCR4 are both significantly elevated in clinical metastatic OS and is associated with resistance to radiotherapy and poor outcome [7-14]. Numerous reports have shown that targeting tumor hypoxia and CXCR4 alone, and in combination with radiotherapy could improve treatment outcome in metastatic OS $[6,7,14-16]$. Prior reports showed that AMD3100 enhances tumor radio sensitivity of other tumor models [22-25]. Our 
Citation: Li S, Li W, Leung C, Kitahara S, Liu Y, et al. (2018) Combined Angiotensin Receptor Blocker Losartan and the CXCR4 Inhibitor AMD3100 Increases the Efficacy of Radiotherapy in a Metastatic Osteosarcoma Mouse Model. J Cancer Sci Ther 10: 371-378. doi: 10.4172/19485956.1000570

studies in OS suggest that combining a CXCR4 inhibitor (AMD3100) with radiotherapy may delay Os-P0107 tumor growth compared with IR alone, although the magnitude of the additional delay did not achieve statistical significance. Nevertheless, triple combination treatment of losartan and AMD3100 with radiotherapy in Os-P0107 isografted tumors significantly delayed local tumor growth when compare to IR alone, decreases distant metastases, and increases host survival. This combination therapeutic effect was most likely due to the targeting of tumor solid stress by losartan, resulting in decompressed tumor vessels and increased vascular perfusion, and AMD3100 delivery to tumors. Our finding may provide a novel therapeutic strategy to complement current metastatic OS treatments [45].

\section{Conclusion}

In summary, using an established mouse model of spontaneously metastatic OS with CXCR4 overexpression and collagen-rich stromal component, we show that combining losartan and AMD3100 with radiotherapy can significantly enhance local tumor growth delay, decrease metastases, and increase host survival. These therapeutic effects are most likely due to the targeting both of tumor hypoxia and CXCR4 by losartan and AMD3100 with local irradiation. Our finding strongly suggests that losartan, and AMD3100 with radiotherapy could be a potential new strategy for clinical metastatic OS treatment.

\section{Acknowledgements}

We thank the Cox-7 animal care takers for their excellent support; Sylvie Roberge, Hao Liu, and Shanmin Chin for their technical advice and support; Dr. Vikash V. Chauhan for helpful input with the study design and the manuscript preparation. This work is supported in part by US NIH National Cancer Institute grants (P01CA080124 to D.F, D.G.D. and R.K.J.; R01-CA096915 to D.F.; R01CA159258 and R41-CA213678 to D.G.D.; R01-CA129371, R01-CA208205 U01-CA 224348, and Outstanding Investigator Award R35-CA197743, and P50CA165962 to R.K.J; and NCI/Federal Share Proton Beam Program Income to P.H.); and Alex's Lemonade Stand Foundation to S.K.

\section{References}

1. Guijarro MV (2014) Osteosarcoma: Mouse models, cell of origin and cancer stem cell. Postdoc J 2: 19-30.

2. Gill J, Ahluwalia MK, Geller D, Gorlick R (2013) New targets and approaches in osteosarcoma. Pharmacol Ther 137: 89-99.

3. Scott MC, Tomiyasu H, Garbe JR, Cornax I, Amaya C, et al. (2016) Heterotypic mouse models of canine osteosarcoma recapitulate tumor heterogeneity and biological behavior. Dis Model Mech 9: 1435-1444.

4. Isakoff MS, Bielack SS, Meltzer P, Gorlick R (2015) Osteosarcoma: Current treatment and a collaborative pathway to success. J Clin Oncol 33: 3029-3035.

5. Salinas-Souza C, De Oliveira R, Alves MT, Garcia-Filho RJ, Petrilli AS, et al. (2013) The metastatic behavior of osteosarcoma by gene expression and cytogenetic analyses. Hum Pathol 44: 2188-2198.

6. Janeway KA, Walkley CR (2010) Modeling human osteosarcoma in the mouse: From bedside to bench. Bone 47: 859-865.

7. Ritter J, Bielack SS (2010) Osteosarcoma. Ann Oncol 21: 320-325.

8. Guo M, Cai C, Zhao G, Qiu X, Zhao H, et al. (2014) Hypoxia promotes migration and induces CXCR4 expression via HIF-1 $\alpha$ activation in human osteosarcoma. PLoS One 9: e90518.

9. Setty BA, Jin Y, Houghton PJ, Yeager ND, Gross TG, et al. (2016) Hypoxic proliferation of osteosarcoma cells depend on arginase II. Cell Physiol Biochem 39: 802-813.

10. Liao YX, Zhou CH, Zeng H, Zuo DQ, Wang ZY, et al. (2013) The role of CXCL12-CXCR4/CXCR7 axis in the progression and metastasis of bone sarcomas. Int J Mole Med 32: 1239-1246.

11. Lin F, Zheng SE, Shen Z, Tang LN, Chen P, et al. (2011) Relationships between levels of CXCR4 and VEGF and blood-borne metastasis and survival in patients with osteosarcoma. Med Oncol 28: 649-653.
12. Ma Q, Zhou Y, Ma B, Chen X, Wen Y, et al. (2012) The clinical value of CXCR4 HER2 and CD44 in human osteosarcoma: A pilot study. Oncol Lett 3: 797-801.

13. Oda Y, Yamamoto H, Tamiya S, Matsuda S, Tanaka K, et al. (2006) CXCR4 and VEGF expression in the primary site and the metastatic site of human osteosarcoma: analysis within a group of patients, all of whom developed lung metastasis. Mod Pathol 19: 738-745.

14. Kim SY, Lee CH, Midura BV, Yeung C, Mendoza A, et al. (2008) Inhibition of the CXCR4/CXCL12 chemokine pathway reduces the development of murine pulmonary metastases. Clin Exp Metastasis 25: 201-211.

15. Botter SM, Neri D, Fuchs B (2014) Recent advances in osteosarcoma. Curr Opin Pharmacol 16: 15-23.

16. Heymann D, Redini F (2013) Targeted therapies for bone sarcomas. Bonekey Rep 2: 378.

17. Zhang P, Dong L, Yan K, Long H, Yang TT, et al. (2013) CXCR4-mediated osteosarcoma growth and pulmonary metastasis is promoted by mesenchymal stem cell through VEGF. Oncol Rep 30: 1753-1761.

18. Li JK, Yu L, Shen Y, Zhou LS, Wang YC, et al. (2008) Inhibition of CXCR4 activity with AMD3100 decreases invasion of human colorectal cancer cell in vitro. World J Gastroenterol 14: 2308-2313.

19. Jeong WJ, Choi IJ, Park MW, An SY, Jeon EH, et al. (2014) CXCR4 antagonist inhibits perineural invasion of adenoid cystic carcinoma. J Clin Pathol 67: 992-998.

20. Chen Y, Ramjiawan RR, Reiberger T, Ng MR, Hato T, et al. (2015) CXCR4 inhibition in tumor microervironment facilitates anti-PD-1 immunotherapy in sorafenib-treted HCC in mice. Hepatology 61: 1591-1602.

21. Hiratsuka S, Duda DG, Huang Y, Goel S, Sugiyama T, et al. (2011) C-X-C receptor type 4 promotes metastasis by activating p38 mitogen-activated protein kinase in myeloid differentiation antigen (Gr-1)-positive cells. Proc Nat Acad Sci USA 108: 302-307.

22. Domanska UM, Boer JC, Timmer-Bosscha H, Van Vugt MA, Hoving HD, et al. (2014) CXCR4 inhibition enhances radiosensitivity, while inducing cancer cell mobilization in a prostate cancer mouse model. Clin Exp Metastasis 31 : 829-839.

23. Kioi M, Vogel H, Schultz G, Hoffman RM, Harsh GR, et al. (2010) Inhibition of vasculogenesis, but not angiogenesis, prevents the recurrence of glioblastoma after irradiation in mice. J Clin Invest 120: 694-705.

24. Kozin SV, Kamoun WS, Huang Y, Dawson MR, Jain RK, et al. (2010) Recruitment of myeloid but not endothelial precursor cells facilitates tumor regrowth after local irradiation. Cancer Res 70: 5679-5685.

25. Duda DG, Kozin SV, Kirkpatrick ND, Xu L, Fukumura D, et al. (2011) CXCL12 (SDF1alpha)-CXCR4/CXCR7 pathway inhibition: An emerging sensitizer for anti-cancer therapies?. Clin Cancer Res 17: 2074-2080.

26. Ek ET, Dass CR, Choong PF (2006) Commonly used mouse models of osteosarcoma. Critical Rev in Oncol/Hematol 60: 1-8.

27. Fan TM (2010) Animal models of osteosarcoma. Expert Rev Anticancer Ther 10: $1327-1338$.

28. Huang P, Mckee TD, Jain RK, Fukumura D (2005) Green fluorescent protein (GFP)-expressing tumor model derived from a spontaneous osteosarcoma in a vascular endothelial growth factor (VEGF)-GFP transgenic mouse. Comp Med 55: 236-243.

29. Nia HT, Liu H, Seano G, Datta M, Jones D, et al. (2016) Solis stress and elastic energy as measures of tumor mechanopathology. Nat Biomed Eng 1: 0004.

30. Chauhan VP, Martin JD, Liu H, Lacorre DA, Jain SR, et al. (2013) Angiotensin inhibition enhances drug delivery and potentiates chemotherapy by decompressing tumor blood vessels. Nat Commun 4: 2516.

31. Pinter M, Jain RK (2017) Targeting the renin-angiotensin system to improve cancer treatment: implications for immunotherapy. Sci Transl Med 9 : EAAN5616.

32. Pinter M, Kwanten WJ, Jain RK (2018) Renin-angiotensin system inhibitors to mitigate cancer treatment-related adverse events. Clin Cancer Res 24: 38033812.

33. Habashi JP, Judge DP, Holm TM, Cohn RD, Loeys BL, et al. (2006) Losartan, an AT1 antagonist, prevents aortic aneurysm in a mouse model of Marfan syndrome. Science 312: 117-121. 
Citation: Li S, Li W, Leung C, Kitahara S, Liu Y, et al. (2018) Combined Angiotensin Receptor Blocker Losartan and the CXCR4 Inhibitor AMD3100 Increases the Efficacy of Radiotherapy in a Metastatic Osteosarcoma Mouse Model. J Cancer Sci Ther 10: 371-378. doi: 10.4172/19485956.1000570

34. Cohn RD, Van Erp C, Habashi JP, Soleimani AA, Klein EC, et al. (2007) Angiotensin II type 1 receptor blockade attenuates TGF- $\beta$-induced failure of muscle regeneration in multiple myopathic states. Nat Med 13: 204-210.

35. Gerweck LE, Vijayappa S, Kurimasa A, Ogawa K, Chen DJ (2006) Tumor cell radiosensitivity is a major determinant of tumor response to radiation. Cancer Res 66: 8352-8355.

36. Huang P, Westmoreland SV, Jain RK, Fukumura D (2011) Spontaneous nonthymic tumors in SCID mice. Comp Med 61: 227-234.

37. Huang P, Allam A, Taghian A, Freeman J, Duffy M, et al. (1995) Growth and metastatic behavior pf five human glioblastomas compared with nine other histological type of human tumor xenografts in SCID mice. J Neurosurg 83: 308-315.

38. Huang Y, Yuan J, Righi E, Kamoun WS, Ancukiewicz M, et al. (2012) Vascular normalizing doses of antiangiogenic treatment reprogram the immunosuppressive tumor microenvironment and enhance immunotherapy. Proc Natl Acad Sci USA 109: 17561-17566.

39. Gerweck LE, Kornbllth PL, Burlett P, Wang J, Sweigert S (1977) Radiation sensitivity of cultured human glioblastoma cells. Radiology 125: 231-234.
40. Brown LC, Lester RA, Grams MP, Haddock MG, Olivier KR, et al. (2014) Stereotactic body radiotherapy for metastatic and recurrent Ewing sarcoma and osteosarcoma. Sarcoma ID 418270.

41. Mamo T, Mladek AC, Shogren KL, Gustafson C, Gupta SK, et al. (2017) Inhibiting DNA-PKcs radiosensitizes human osteosarcoma cells. Biochem Biophys Res Commun 486: 307-313.

42. Bishop MW, Janeway KA, Gorlick R (2016) Future directions in the treatment of osteosarcoma. Curr Opin Pediatr 28: 26-33.

43. DeLaney TF, Park L, Goldberg SI, Hug EB, Liebsch NJ, et al. (2005) Radiotherapy for local control of osteosarcoma. Int J Radiat Oncol Biol Phys 61: 492-498

44. Lee JA, Paik EK, Seo J, Kim DH, Lim JS, et al. (2016) Radiotherapy and gemcitabine-docetaxel chemotherapy in children and adolescents with unresectable recurrent refractory osteosarcoma. Jpn J Clin Oncol 46: 138-143.

45. Coulson R, Liew SH, Connelly AA, Yee NS, Deb S, et al. (2017) The angiotensin receptor blocker, losartan, inhibits mammary tumor development and progression to invasive carcinoma. Oncotarget 8: 18640-18656. 